\title{
$\boldsymbol{w}$ 変換之拡張 $\boldsymbol{w}$ 変換によるサンプル值系の解析
}

\author{
谷萩 隆柌 \\ 東京工業大学大学院 東京都目黒区大岡山 2-12-1 \\ （昭和 43 年 3 月 22 日受付）
}

\section{Analysis of Sampled-Data Systems Using the $w$-Transform and the Modified $w$-Transform}

Takashi YAHAGI

(Tokyo Institute of Technology, Meguro-ku, Tokyo)

(Received March 22, 1968)

The $w$-transform method and the modified $w$-transform method are developed in this paper. The $w$-transform can be applied to sampled-data systems which have all samplers followed by zeroorder hold devices. The $w$-transform is developed utilizing the change of variable, $w^{-n}=\left(e^{-n s T}-\right.$ $\left.e^{-(n+1) s T}\right) / s$, where $T$ is the sampling period and $s$ is the Laplace transform variable.

The $w$-transform is defined as $W[e(t)]=E(w)=\sum_{n=0}^{\infty} e(n T) w^{-n}$.

As the $w$-transform and the z-transform are the special forms of the Laplace transform, there is no great difference between them. However. the physical meaning of the sampling process using the $w$-transform method is clearer than that of the $z$-transform method.

Using the modified $w$-transform, we can obtain the output response at all times.

An extensive table of $G_{p}(w)$ and $G(w, \mu)$ is presented in the Appendix.

\section{1. ま えがき}

サンプル值系の解析方法は，大別じて時間領域での 解析と Laplace 変换領域での解析とにわけられる. 前者は状態変数を用いて系の特性をサンプリング区間 ごとのベクトル微分方程式で記述して，状態空間法な ぞの方法で解析を行ならので，ディジタル計算機など を利用して定量的なはあくをするのに適している1). 後者では主として Laplace 変換の特殊形である $z$ 変 換や拡張 $z$ 変換が用いられ，定性的なはあくをするの には前者にはないすぐれた面がある゙文。

周知のように， $z$ 変換では周期的に動作する理想的 サンプラによって生じるインパルス列に着目して， $z=e^{s T}$ と和いて解析している。ただし $T$ はサンプリン グ周期である。

しかし，実際のサンプル值系ではサンプラの出力は インパルス列ではないことと，サンプリングパルスに 含末れている高調波成分を除去するために，サンプラ のあとに0 次ホールド回路をつけて用いるのが普通で あることを考慮すれば，サンプラの出力をインパルス
列であると考えて解析する $z$ 変換法よりも, 0 次ホー ルド回路の出力が階段波形であることに着目して，サ ンプラと 0 次ホールド回路をあわせて 1 種のパルス変 調器と考えて，解析を行なら汪らが適切であると考え られる。

これらの理由から， $x^{n}=e^{-n s T} / s$ と抹いて，0次ホ ールド回路の出力に着目して解析を行なら $x$ 変換法が 発表されている5). しかし，0次ホールド回路の出力 に着目するならば，各偝段波形を1つの変数であらわ して解析する法らが有利であると考えられる。これら のことを考慮して，本論文では， $w^{-n}=\left(e^{-n s T}\right.$ $\left.e^{-(n+1) s T}\right) / s$ と和いて解析を行なら $w$ 変換法を提案す る. $w$ 変換を用いれば各サンプリング区間に拉ける0 次ホールド回路の出力応答を求めることができる。さ らに拡張 $w$ 変換は任意の時刻での出力応答が求められ るよらに定義されている。

\section{2. $w$ 変換の定義とその性質}

解析しようとするサンプル值系が，つぎの条件を満 たすものと仮定する。 
1）サンプラは周期的に動作する.

2）サップルされた信号は，初期条件が 0 の線形系 に加わる。

3）サンプラのあとには 0 次ホールド回路がおかれ ていて，その出力は一連の階段波形である.

仮定 1)，2）はz変換を行ならさいにも必要である.

Fig.1に示すサンプリング過程を考学る.

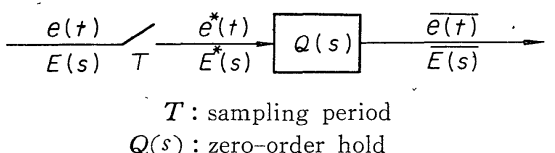

Fig. 1 The sampling process

このとき $\overline{e(t)}$ はつぎのようになる。

$\overline{e(t)}=\sum_{n=0}^{\infty} e(n T)\{u(t-n T)-u(t-\overline{n+1} T)\}$

ただし， $u(t)=$ unit step function である.

$(2 \cdot 1)$ 式を Laplace 変換すれば,

$$
\overline{E(s)}=\sum_{n=0}^{\infty} e(n T) \frac{e^{-n s T}-e^{-(n+1) s T}}{s}
$$

$w^{-n}=\left(e^{-n s T}-e^{-(n+1) s T}\right) / s$ と㧊いて $w$ 変換をつぎのよ うに定義する.

$$
\begin{aligned}
W[e(t)] & =E(w)=\left.\overline{E(s)}\right|_{w^{-n}=\frac{e^{-n s} T-e^{-(n+1) s T}}{s}} \\
& =\sum_{n=0}^{\infty} e(n T) w^{-n}
\end{aligned}
$$

$n$ を任意の整数としてつぎの変数を導入する.

$$
w_{d}{ }^{-n}=e^{-n s T}
$$

$w_{d}$ はパルス移動演算子である.

$m$ を任意の整数としたとき，つぎの等式が成立す る。

$$
\left.\begin{array}{l}
w_{d}{ }^{-m} w^{-n}=w^{-(m+n)} \\
w_{d}^{0}=1
\end{array}\right\}
$$

(2.5) 式から任意の整数 $n$ に対して $w^{-n}$. が定義さ れる。

$$
\begin{aligned}
& \text { 【例】 } e(t)=u(t) \text { のとき, } \\
& E(w)=\sum_{n=0}^{\infty} w^{-n}=w^{0} \sum_{n=0}^{\infty} w_{d}{ }^{-n}=\frac{w}{w_{d}-1} \\
& e(t)=t u(t) \text { のとき, } \\
& E(w)=\sum_{n=0}^{\infty} n T w^{-n}=w^{0} \sum_{n=0}^{\infty} n T w_{d}^{-n}=\frac{T w}{\left(w_{d}-1\right)^{2}}
\end{aligned}
$$

$w$ 変換と $z$ 変換の関係はつぎのようになる。

$(2 \cdot 3)$ 式から，

$$
E(w)=\sum_{n=0}^{\infty} e(n T) w^{-n}=w^{0} \sum_{n=0}^{\infty} e(n T) w_{d}^{-n}
$$

$z$ 変換の定義から,

$$
E(z)=\sum_{n=0}^{\infty} e(n T) z^{-n}
$$

$(2 \cdot 6)$ 式と $(2 \cdot 7)$ 式加,

$$
E(w)=\left.w^{0} E(z)\right|_{z=w_{d}}
$$

つぎに $w$ 変換についての主な定理をあげておく.

【定理 1】【初期値定理】

$E(w)=W[e(t)]$ のとき $\left.\lim _{w \rightarrow \infty} E(w)\right|_{w 0=1}$ が存在すれ ば,

$$
\lim _{n \rightarrow 0} e(n T)=\left.\lim _{w \rightarrow \infty} E(w)\right|_{w^{0}=1}
$$

【証明】 $w$ 変換の定義加ら，

$$
\begin{aligned}
& E(w)=\sum_{n=0}^{\infty} e(n T) w^{-n}=w^{0} \sum_{n=0}^{\infty} e(n T) w_{d}-n \\
&\left.\therefore \quad \lim _{w \rightarrow \infty} E(w)\right|_{w^{0}=1}=\lim _{w_{d \rightarrow \infty}} \sum_{n=0}^{\infty} e(n T) w_{d}{ }^{-n} \\
&=\lim _{n \rightarrow 0} e(n T)
\end{aligned}
$$

【例】 $e(t)=t u(t)$ のとき' $E(w)=\frac{T w}{\left(w_{d}-1\right)^{2}}=$ $\frac{T w\left(w^{0}\right)^{2}}{\left(w-w^{0}\right)^{2}}$ であるから, 初期值定理を用いると,

$$
\lim _{n \rightarrow 0} e(n T)=\lim _{w \rightarrow \infty} \frac{T w}{(w-1)^{2}}=0
$$

【定理 2】[最終值定理】

$E(w)=W[e(t)]$ のとき, 最終值が存在すれば，

$$
\lim _{n \rightarrow \infty} e(n T)=\left.\lim _{w \rightarrow 1} \frac{w-1}{w} E(w)\right|_{w^{0}=1}
$$

【証明】 $w$ 変換の定義から，

$$
\begin{gathered}
W[e(t)]=E(w)=\sum_{n=0}^{\infty} e(n T) w^{-n} \\
W[e(t+T)]=\sum_{n=0}^{\infty} e(\overline{n+1} T) w^{-n} \\
=w_{d} E(w)-e(0) w \\
\therefore W[e(t+T)-e(t)]=\left(w_{d}-1\right) E(w)-e(0) w \\
w^{0}=1 \text { とすれば } w_{d}=w \text { となるから, } \\
\left.W[e(t+T)-e(t)]\right|_{w^{0}=1} \\
=\left.(w-1) E(w)\right|_{w^{0}=1}-e(0) w . \\
\left.\therefore \lim _{w \rightarrow 1} W[e(t+T)-e(t)]\right|_{w^{0}=1} \\
=\left.\lim _{w \rightarrow 1}(w-1) E(w)\right|_{w^{0}=1}-e(0)
\end{gathered}
$$

いっぽら，

$$
\begin{aligned}
& W[e(t+T)-e(t)] \\
& \quad=\lim _{n \rightarrow \infty} \sum_{k=0}^{n}[e(\overline{k+1} T)-e(k T)] w^{-k}
\end{aligned}
$$

最終值が存在すれば，

$$
\begin{aligned}
& \left.\lim _{w \rightarrow 1} W[e(t+T)-e(t)]\right|_{w 0}=1 \\
& \quad=\left.\lim _{w \rightarrow 1} \lim _{n \rightarrow \infty} \sum_{k=0}^{n}[e(\overline{k+1} T)-e(k T)] w^{-k}\right|_{w 0}=1 \\
& \quad=\lim _{w_{d} \rightarrow 1} \lim _{n \rightarrow \infty} \sum_{k=0}^{n}[e(\overline{k+1} T)-e(k T)] w_{d}^{-k} \\
& \quad=\lim _{n \rightarrow \infty} \lim _{w_{d} \rightarrow 1} \sum_{k=0}^{n}[e(\overline{k+1} T)-e(k T)] w_{d}-k \\
& =\lim _{n \rightarrow \infty} e(\overline{n+1} T)-e(0)
\end{aligned}
$$

$(2 \cdot 11)$ 式と $(2 \cdot 12)$ 式から， 


$$
\begin{aligned}
& \left.\lim _{n \rightarrow \infty} e \overline{(n+1} T\right)=\left.\lim _{w \rightarrow 1}(w-1) E(w)\right|_{w 0=1} \\
\therefore & \lim _{n \rightarrow \infty} e(n T)=\lim _{w \rightarrow 1} \frac{w-1}{w}-\left.E(w)\right|_{w^{0}=1}
\end{aligned}
$$

【例】 $e(t)=e^{-a T} u(t)$ のとき $(a>0)$ ，

$$
E(w)=\frac{w}{w_{d}-e^{-a T-}}=\frac{w w^{0}}{w-e^{-a T} w^{0}} \text { であるから， }
$$

最終值定理を用いれば，

$$
\lim _{n \rightarrow \infty} e(n T)=\lim _{w \rightarrow 1} \frac{w-1}{w} \frac{w}{w-e^{-a T}}=0
$$

【定理 3】 $a$ を独立变数として，

$E(w, a)=W[e(t, a)]$ と叔いたとき，

$$
\frac{\partial}{\partial a} E(w, a)=W\left[\frac{\partial}{\partial a} e(t, a)\right]
$$

\section{【証明】}

$$
\begin{aligned}
& W\left[\frac{\partial}{\partial a} e(t, a)^{\cdot}\right]=\sum_{n=0}^{\infty}\left[-\frac{\partial}{\partial a}-e(n T, a)\right] w^{-n} \\
& =\frac{\partial}{\partial a} \sum_{n=0}^{\infty} e(n T, a) w^{-n}=\frac{\partial}{\partial a} E(w, a)
\end{aligned}
$$

【定理 4】 $a$ を独立変数， $\alpha, \beta$ を定数としたとき，

$$
\int_{\alpha}^{\beta} E(w, a) d a=W\left[\int_{\alpha}^{\beta} e(t, a) d a\right]
$$

【定理 5】 $a$ を独立変数， $b$ を定数としたとき，

$$
\lim _{a \rightarrow b} E(w, a)=W\left[\lim _{a \rightarrow b} e(t, a)\right]
$$

\section{3. 逆 $w$ 变換の求め方}

$E(w)$ があた兄られたとき，これを逆変換して $e(n T)$ を求める方法を述べる.

$w$ 変換の定義から,

$$
\begin{aligned}
E(w) & =\sum_{n=0}^{\infty} e(n T) w^{-n}=w^{0} \sum_{n=0}^{\infty} e(n T) w_{d}^{-n} \\
\therefore \frac{E(w)}{w^{0}} & =\sum_{n=0}^{\infty} e(n T) w_{d}^{-n} \\
& =e(0)+e(T) w_{d}^{-1}+\cdots+e(n T) w_{d}{ }^{2 n}+\cdots,
\end{aligned}
$$

両辺に $w_{d}^{n-1}$ をかけると，

$$
\begin{gathered}
\frac{E(w)}{w^{0}} w_{d}^{n-1}=e(0) w_{d}^{n-1}+e(T) w_{d}^{n-2}+\cdots \\
\cdots+e(n T) w_{d}^{-1}+\cdots,
\end{gathered}
$$

いま積分路 $\Gamma$ を， $w_{d}$ 平面に执いて $\frac{E(w)}{w^{0}} w_{d}^{n-1}$ の 無限遠点を除いた特異点を含をようにとり，反時計方 向に積分を行ならものとすると，nが負でない整数の とき，(3·1)式を積分して，

$$
\begin{aligned}
e(n T) & =\frac{1}{2 \pi j} \int_{\Gamma} \frac{E(w)}{w^{0}} w_{d}^{n-1} d w_{d} \\
& =\Gamma \text { に打をる } \frac{E(w)}{w^{0}} w_{d}^{n-1} \text { の留数の和 }
\end{aligned}
$$

被積分項が点 $a$ 飞 $k$ 次の多重極をむつ場合，

$$
\operatorname{Res} e_{k}(n T)=\frac{1}{(k-1) !} \frac{d^{k-1}}{d w_{d}^{k-1}}
$$

$$
\left.\cdot\left[\frac{E(w)}{w^{0}}\left(w_{d}-a\right)^{k} w_{d}^{n-1}\right]\right|_{w_{d} \rightarrow a}
$$

【例】 $E(w)=\frac{T w}{\left(w_{d}-1\right)^{2}}$ のとき，

$$
\begin{aligned}
e(n T) & =\frac{1}{2 \pi j} \int_{\Gamma} \frac{w_{d}^{n-1}}{w^{0}} \frac{T w}{\left(w_{d}-1\right)^{2}} d w_{d} \\
& =\frac{1}{2 \pi j} \int_{\Gamma} \frac{T w_{d}^{n}}{\left(w_{d}-1\right)^{2}} d w_{d}
\end{aligned}
$$

(3.3) 式で $k=2, a=1$ と执いて,

$$
e(n T)=\left.\frac{d}{d w_{d}}\left(T w_{d}^{n}\right)\right|_{w_{d} \rightarrow 1}=n T
$$

$z$ 変換の場合々同じく $w$ 変換の場合にも無限級数法が 適用できる。

これを用いて上の例についてといてみると，

$$
\begin{aligned}
& E(w)=\frac{T w}{\left(w_{d}-1\right)^{2}}=T w^{-1}+2 T w^{-2}+3 T w^{-3}+\cdots \\
& \quad=\sum_{n=0}^{\infty} n T w^{-n} \\
& \therefore \quad e(n T)=n T
\end{aligned}
$$

このように $w_{d}$ 平面に打ける $\frac{E(w)}{w^{0}} w_{d}^{n-1}$ の留数を 計算する方法, あるいは $E(w)$ を無限級数汇展 開す る方法を用いることにより $e(n T)$ が求められる.

\section{4. $\boldsymbol{w}$ 変換によるサンプル値系の解析}

サンプル值系の解析に $w$ 変換を商用する方法につい て述べる。

Fig.2 に示す開ループ系を考光る。

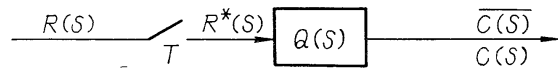

Fig. 2 Open loop sampled-data system

$$
\begin{aligned}
& \overline{C(s)}=R^{*}(s) Q(s)=\overline{R(s)} \\
& \therefore \quad C(w)=R(w)
\end{aligned}
$$

Fig. 3 飞示す開ループ系を考光る.

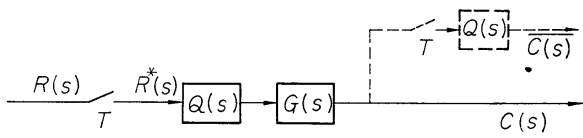

Fig. 3 Open loop sampled-data system

$$
\begin{aligned}
& C(s)=R^{*}(s) Q(s) G(s) \\
& \overline{C(s)}=R^{*}(s)(G(s) Q(s))^{*} Q(s) \\
&=\overline{R(s)}(G(s) Q(s))^{*} \\
& \therefore \quad C(w)=R(w) G_{p}(w)
\end{aligned}
$$

ここで,

$$
\begin{aligned}
G_{p}(w) & =W\left[(G(s) Q(s))^{*}\right] \\
& =\left(1-w_{a}^{-1}\right) W\left[\left(\frac{G(s)}{s}\right)^{*}\right] \\
& =\frac{1-w_{d}^{-1}}{w^{0}}-W\left[\frac{G(s)}{s}\right]=\frac{w-w^{0}}{w w^{0}} W\left[\frac{G(s)}{s}\right]
\end{aligned}
$$


Fig.4 に示すフィードバック系を考光る.

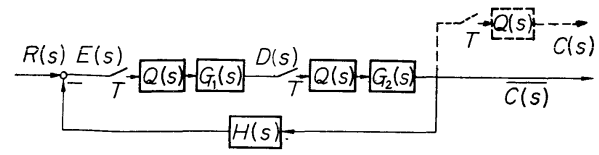

Fig. 4 Feed back sampled-data system

$$
\begin{aligned}
& E(s)=R(s)-D^{*}(s) Q(s) G_{2}(s) H(s) \\
& D(s)=E^{*}(s) Q(s) G_{1}(s) \\
& C(s)=D^{*}(s) Q(s) G_{2}(s) \\
& \therefore \quad \overline{C(s)}=C^{*}(s) Q(s) \\
&=\frac{\overline{R(s)}\left(G_{1}(s) Q(s)\right)^{*}\left(G_{2}(s) Q(s)\right)^{*}}{1+\left(G_{1}(s) Q(s)\right)^{*}\left(G_{2}(s) H(s) Q(s)\right)^{*}} \\
& \therefore \quad C(w)=\frac{R(w) G_{1 p}(w) G_{2 p}(w)}{1+G_{1 p}(w)\left(G_{2} H\right)_{p}(w)} \\
& こ こ て ゙ \\
& G_{1 p}(w)=W\left[\left(G_{1}(s) Q(s)\right)^{*}\right] \\
& G_{2 p}(w)=W\left[\left(G_{2}(s) Q(s)\right)^{*}\right] \\
&\left(G_{2} H\right)_{p}(w)=W\left[\left(G_{2}(s) H(s) Q(s)\right)^{*}\right]
\end{aligned}
$$

【例】簡単な例として, Fig.5 の系で $G(s)=1(s+)$ $1, R(s)=1 / s$ とした之き $C(w), C(n T)$ はつぎのよ うになる。

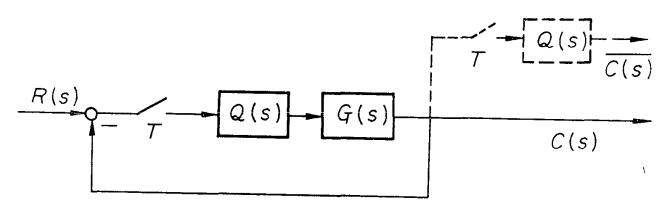

Fig. 5 Illustrative example

$$
\begin{aligned}
& C(w)=\frac{R(w) G_{p}(w)}{1+G_{p}(w)} \\
& G_{p}(w)=W[(G(s) Q(s)) *]=\frac{\left(1-e^{-T}\right) w^{0}}{w-e^{-T} w^{0}}=\frac{1-e^{-T}}{w_{d}-e^{-T}} \\
& R(w)=\frac{w}{w_{d}-1} \\
& \therefore \quad C(w)=\frac{\left(1-e^{-T}\right) w}{\left(w_{d}-1\right)\left(w_{d}+1-2 e^{-T}\right)} \\
& C(n T)=\frac{1}{2 \pi j} \int_{\Gamma} \frac{\left(1-e^{-T}\right) w_{d}^{n}}{\left(w_{d}-1\right)\left(w_{d}+1-2 e^{-T}\right)} d w_{d} \\
& \quad=\frac{1}{2}-\frac{1}{2}\left(2 e^{-T}-1\right)^{n}
\end{aligned}
$$

\section{5. 拡張 $\boldsymbol{w}$ 变換の定義とその性質}

拡張 $w$ 変換をつぎのように定義する.

$W_{\mu}[e(t)]=W[e(t+\mu T)]$

$$
=E(w, \mu)=\sum_{n=0}^{\infty} e(n, \mu) w^{-n}
$$

ここで,

$$
e(n, \mu)=e(\overline{n+\mu} T), \quad 0 \leq \mu \leq 1
$$

$(5 \cdot 1)$ 式と $(2 \cdot 3)$ 式から，

$$
\lim _{\mu \rightarrow 0} E(w, \mu)=E(w)
$$

$E(w, \mu)$ を求めるために, Fig.6 に示すような仮 想進夕時間 $\mu T$ を導入したサンプリング過程を考え る.

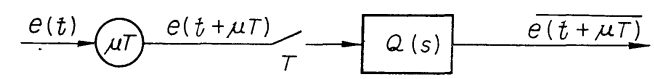

Fig. 6 The sampling process

このとき，

$$
\begin{aligned}
& E(w, \mu)=\left.\mathcal{L}\left[e(t+\mu T) \delta_{T}(t)\right] Q(s)\right|_{w^{-n}=\frac{e^{-n s} T-e^{-(n+1) s} T}{s}} \\
& \mathcal{L}[e(t+\mu T)]=E(s) e^{\mu s T} \\
& \mathcal{L}\left[\delta_{T}(t)\right]=\frac{1}{1-e^{-s T}} \\
& \therefore \quad E(w, \mu)=\frac{1}{2 \pi j} \int_{C-j \infty}^{C+j \infty} E(p) e^{\mu p T} \frac{w}{w_{d}-e^{p T}} d p \\
& =\sum_{E(s) \text { 極 }} E(s) \text { の留数 } \times e^{\mu S T} \frac{w}{w_{d}-e^{s T}}
\end{aligned}
$$

$(5 \cdot 1)$ 式あるいは $(5 \cdot 4)$ 式から $E(w, \mu)$ が求められ， $(5 \cdot 2)$ 式の関係を利用すれば $E(w)$ b求めることがで きる. 伝達関数 $G(s)$ があた兄られた場合には， $(5 \cdot 4)$ 式の右辺の $E(s)$ のかわりに $G(s)$ を代入すれ ば, $G(w, \mu)$ が求められる.

【例】 $e(t)=t u(t)$ のとき，

$(5 \cdot 1)$ 式あるいは $(5 \cdot 4)$ 式から，

$$
E(w, \mu)=-\frac{\mu T w}{w_{d}-1}+\frac{T w}{\left(w_{d}-1\right)^{2}}
$$

まな $(5 \cdot 2)$ 式から，

$$
E(w)=\lim _{\mu \rightarrow 0} E(w, \mu)=\frac{T w}{\left(w_{d}-1\right)^{2}}
$$

これは (2.3) 式を用いて得られた結果と一致してい る.つぎに拡張 $w$ 変換の主な定理を述べる.ただし $w$ 変換のときと大体同様にして証明することができる場 合には証明を省略する。

\section{【定理 6】【初期䛧定理】}

$E(w, \mu)=W_{\mu}[e(t)]$ のとき $\left.\lim _{\substack{w \rightarrow \infty \\ \mu \rightarrow 0}} E(w, \mu)\right|_{w^{0}=1}$ が存 在すれば，

$$
\lim _{\substack{n \rightarrow 0 \\ \mu \rightarrow 0}} e(n, \mu)=\left.\lim _{\substack{w \rightarrow \infty \\ \mu \rightarrow 0}} E(w, \mu)\right|_{w^{0}}=1
$$

【定理 7】【最終值定理】

$E(w, \mu)=W_{\mu}[e(t)]$ のとき, 最終值が存在すれば,

$$
\lim _{n \rightarrow \infty} e(n, \mu)=\left.\lim _{w \rightarrow 1} \frac{w-1}{w} E(w, \mu)\right|_{w 0=1}
$$

【例】 $e(t)=e^{-a t} u(t)$ のとき $(a>0)$, 


$$
E(w, \mu)=\frac{e^{-\mu a T} w}{w_{d}-e^{-a T}}=\frac{e^{-\mu a T} w^{0} w}{w-e^{-a T} w^{0}}
$$

初期值定理を用いれば，

$$
\lim _{\substack{w \rightarrow 0 \\ \mu \rightarrow 0}} e(n, \mu)=\lim _{\substack{n \rightarrow \infty \\ \mu \rightarrow 0}} \frac{e^{-\mu a T} w}{w-e^{-a T}}=1
$$

最終值定理を用いれば，

$$
\lim _{n \rightarrow \infty} e(n, \mu)=\lim _{w \rightarrow 1} \frac{w-1}{w} \frac{e^{-\mu a T} w}{w-e^{-a T}}=0
$$

【定理 8】〔 $\mu$ による微分定理】

$E(w, \mu)=W_{\mu}[e(t)]$ のとき，

$$
\frac{\partial}{\partial \mu} E(w, \mu)=W_{\mu}\left[\frac{\partial}{\partial \mu} e(n, \mu)\right]
$$

【定理 9】〔 $\mu$ による積分定理〕

$E(w, \mu)=W_{\mu}[e(t)]$ のとき，

$$
\int_{\alpha}^{\beta} E(w, \mu) d \mu=W_{\mu}\left[\int_{\alpha}^{\beta} e(n, \mu) d \mu\right]
$$

ただし $\alpha, \beta$ は $0 \leq \alpha \leq \beta \leq 1$ を満たす定数である.

【定理 10】 $a$ を定数として,

$W_{\mu}[E(s)]=w^{0} E\left(w_{d}, \mu\right)$ と扔いたとき，

$W_{\mu}[E(s+a)]=w^{0} e^{-\mu a T} E\left(e^{a T} w_{d}, \mu\right)$

\section{【証明】}

$$
\begin{aligned}
W_{\mu}[E(s)] & =w^{0} E\left(w_{d}, \mu\right)=\sum_{n=0}^{\infty} e(n, \mu) w^{-n} \\
& =w^{0} \sum_{n=0}^{\infty} e(n, \mu) w_{d}^{-n}
\end{aligned}
$$$$
\therefore \quad W_{\mu}[E(s+a)]=\sum_{n=0}^{\infty} e^{-(n+\mu) a T} e(n, \mu) w^{-n}
$$$$
=w^{0} e^{-\mu a T} \sum_{n=0}^{\infty} e(n, \mu)\left(e^{a T} w_{d}\right)^{-n}
$$$$
=w^{0} e^{-\mu a T} E\left(e^{a T} w_{d}, \mu\right)
$$

【例】 $W_{\mu}[E(s)]=\frac{\mu T w}{w_{d}-1}+\frac{T w}{\left(w_{d}-1\right)^{2}}$ のとき，

$$
E\left(w_{d}, \mu\right)=\frac{\mu T w_{d}}{w_{d}-1}+\frac{T w_{d}}{\left(w_{d}-1\right)^{2}}
$$

$\therefore \quad W_{\mu}[E(s+a)]=\frac{\mu T e^{-\mu a T} w}{w_{d}-e^{-a T}}+\frac{T e^{-(1+\mu) a T} w}{\left(w_{d}-e^{-a T}\right)^{2}}$

\section{6. 逆拡張 $\boldsymbol{w}$ 変換の求め方}

逆 $w$ 変換の場合と同様にして，

$$
e(n, \mu)=\frac{1}{2 \pi j} \int_{\Gamma} \frac{E(w, \mu)}{w^{0}} w_{d}^{n-1} d w_{d}
$$

ここで $\Gamma$ は被積分項の無限遠点を除いたすべての特 異点をらくむようにとり，反時計方向に積分を行なら ものとする。

妾 0 から 1 までの間で変化させれば，任意の時刻 での応答を求めることができる。

系のインパルス応答が各サンプリング時点で不連続 の場合には, $(6 \cdot 1)$ 式で $\mu \rightarrow 0$ とすればサンプリング

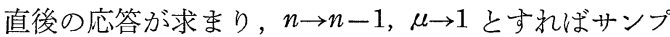

リング直前の応答が求められる。

すなわち，

$$
\left.\begin{array}{l}
\lim _{\mu \rightarrow 0} e(n, \mu)=e\left(n T^{+}\right) \\
\lim _{\mu \rightarrow 1} e(n-1, \mu)=e\left(n T^{-}\right)
\end{array}\right\}
$$

【例】 $e(t)=t u(t)$ のとき,

(6.1)式を用いると,

$$
E(w, \mu)=\frac{\mu T w}{w_{d}-1}+\frac{T w}{\left(w_{d}-1\right)^{2}}
$$

$$
\begin{aligned}
e(n, \mu) & =\frac{1}{2 \pi j} \int_{\Gamma}\left\{\frac{\mu T w_{d}{ }^{n}}{w_{d}-1}+\frac{T w_{d}{ }^{n}}{\left(w_{d}-1\right)^{2}}\right\} d w_{d} \\
& =(n+\mu) T
\end{aligned}
$$

\section{7. 拡張 $\boldsymbol{w}$ 変換によるサンプル值系の解析}

$w$ 変換によるサンプル值系の解析と対応させる意味 で，4. で示した系について考觉る。

Fig. 2 の開ループ系の場合，

$$
\begin{aligned}
& C(s)=R^{*}(s) Q(s)=\overline{R(s)} \\
& \therefore \quad C(w, \mu)=R(w)
\end{aligned}
$$

Fig. 3 の開ループ系の場合,

$$
C(s)=R^{*}(s) Q(s) G(s)
$$

$W_{\mu}\left[R^{*}(s)\right]=W\left[R^{*}(s)\right]=\frac{R(w)}{w^{0}}$ であるから，

$$
C(w, \mu)=\frac{R(w)}{w^{0}} G_{p}(w, \mu)
$$

ここで,

$$
G_{p}(w, \mu)=W_{\mu}[G(s) Q(s)]
$$

Fig. 4 のフィードバック系の場合,

$$
\begin{array}{r}
C(s)=\frac{R^{*}(s)\left(G_{1}(s) Q(s)\right) * Q(s) G_{2}(s)}{1+\left(G_{1}(s) Q(s)\right)^{*}\left(G_{2}(s) H(s) Q(s)\right)^{*}} \\
\therefore \quad C(w, \mu)=\frac{R(w)}{w^{0}} \frac{G_{1 p}(w) G_{2 p}(w, \mu)}{1+G_{1 p}(w)\left(G_{2} H\right)_{p}(w)}
\end{array}
$$

8. あとがき

$w$ 変換法および拡張 $w$ 変換法を提案し，これらを用 いてサンプル值系の解析を行なら方法を示した．本論 文では $w^{-n}=\left(e^{-n s T}-e^{-(n+1) s T}\right) / s$ とおいて $w$ 変換を 定義している. 定義加らもわかるように，w变換はサ ンプラのあとに0 次ホールド回路がある場合のサンプ ル值系の解析に適用できる.しかし実際のサンプル值 系では，サンプリングパルスに含まれている高調波成 分を除去するために，サンプラのあとに0次ホールド 回路をつけるのが普通であるので，この条件はそれほ ぞ厳しいものではない。

拡張 $w$ 変換を用いれば，任意の時刻での出力応答を 求めることができる.

$w$ 変換法は $z$ 変換法に対応するものであるが，これ らを比較すればつぎのよらになる。 
$z$ 変換では理想的サンプラの出力に着目しているの で，理論上はサンプラの出力は高さが無限大で面積が 害際の出力に等しいインパルス列である。

しかし物理的に実現可能なサンプラの出力は，微小 な有限幅をもったパルス列であり，パルス列の高さが 出力の大きさをあらわしている。ここに $z$ 変換の理論 と実際との間に gap があり，これが $z$ 変換法がすっ きりしない理由であると思われる。

$w$ 変換では，たとえば $(2 \cdot 3)$ 式を又ればわかるよう に，0次ホールド回路の出力に着目しているので階段 波形の高さが出力の大ささをあらわして拉り， $z$ 変換 の場合のような不自然さはない。

また $w$ 変換法と $x$ 変換法を比べると, $w$ 変換法の注 らが式の操作が簡単である点などが有利である.

$w$ 変換では变数として $w$ の症かにパルス移動演算子 $w_{d}$ が導入されているが，これは解析を簡単にする ために導入したもので， $w_{d}=w / w^{0}$ の関係を用いれば を $w_{d}$ 消去することがでさる.
本方法を使用するさいの便宜のため，各種の $G(s)$ に対する $G_{p}(w)$ と $G(w, \mu)$ を付録江して和く.

$(5 \cdot 2)$ 式の関係を利用すれば $G(w)$ もすぐに求めら れる。これらの各式は $z$ 変換および拡張 $z$ 変換の場合 とだいたい同程度の複雑さである。

敃わりに，いつもあたたかい御指導と御激励をして くださいます，東京工業大学の粟屋 潔教授ならびに 今井 聖助教授に深く感謝いたします.

\section{文献}

1) J. T. Tou : "Modern Control Theory", McGraw-Hill (1964)

2) E. I. Jury : "Sampled-Data Control Systems", John Wiley \& Sons (1958)

3) J. T. Tou : "Digital and Sampled-Data Control Systems", McGraw-Hill (1959)

4) Л.Т.Кузин : "Расчет и Проектирование Дискретных Систем Управления”, Машгиз (1962)

5) W.L. McDaniel, C. L. Phillips : "The X-Transform and its Use in Sampled-Data System Analysis", J. Franklin Inst. 284-1, pp. 39/55 (1967)

付

録

\begin{tabular}{|c|c|c|}
\hline$G(s)$ & $G_{p}(w)$ & $G(w, \mu)$ \\
\hline$\frac{1}{s}$ & $\frac{T w^{0}}{w-w^{0}}$ & $\frac{w}{w_{d}-1}$ \\
\hline$\frac{1}{s^{2}}$ & $\frac{T^{2} w^{0}\left(w+w^{0}\right)}{2\left(w-w^{0}\right)^{2}}$ & $\frac{\mu T w}{w_{d}-1}+\frac{T w}{\left(w_{d}-1\right)^{2}}$ \\
\hline$\frac{1}{s^{n}}$ & $\lim _{a \rightarrow 0} \frac{(-1)^{n-1}}{(n-1) !} \frac{\partial^{n-1}}{\partial a^{n-1}}\left[\frac{1}{a} \frac{\left(1-e^{-a T}\right) w^{0}}{w-e^{-a T} w^{0}}\right]$ & $\lim _{a \rightarrow 0} \frac{(-1)^{n-1}}{(n-1) !} \frac{\partial^{n-1}}{\partial a^{n-1}} \frac{e^{-\mu a T} w}{w_{d}-e^{-a T}}$ \\
\hline$\frac{1}{s+a}$ & $\frac{1}{a} \frac{\left(1-e^{-a T}\right) w^{0}}{w-e^{-a T} w^{0}}$ & $\frac{e^{-\mu a T} w}{w_{d}-e^{-a T}}$ \\
\hline$\frac{1}{(s+a)^{2}}$ & $\frac{1}{a^{2}} \frac{\left(1-e^{-a T}\right) w^{0}}{w-e^{-a T} w^{0}}-\frac{1}{a} \frac{T e^{-a T} w^{0}\left(w-w^{0}\right)}{\left(w-e^{-a T} w^{0}\right)^{2}}$ & $\frac{\mu T e^{-\mu a T} w}{w_{d}-e^{-a T}}+\frac{T e^{-(1+\mu) a T} w}{\left(w_{d}-e^{-a T}\right)^{2}}$ \\
\hline$\frac{1}{(s+a)^{n}}$ & $\frac{(-1)^{n-1}}{(n-1) !} \frac{\partial^{n-1}}{\partial a^{n-1}}\left[\frac{1}{a} \frac{\left(1-e^{-a T}\right) w^{0}}{w-e^{-a T} w^{0}}\right]$ & $\frac{(-1)^{n-1}}{(n-1) !} \frac{\partial^{n-1}}{\partial a^{n-1}} \frac{e^{-\mu \xi a T} w}{w_{d}-e^{-a T}}$ \\
\hline$\frac{1}{s(s+a)}$ & $-\frac{1}{a^{2}}+\frac{T}{a} \frac{w^{0}}{w-w^{0}}+\frac{1}{a^{2}} \frac{w-w^{0}}{w-e^{-a T} w^{0}}$ & $\frac{1}{a} \frac{w}{w_{d}-1}-\frac{1}{a} \cdot \frac{e^{-\mu a T} w}{w_{d}-e^{-a T}}$ \\
\hline$\frac{1}{(s+a)(s+b)}$ & $\frac{1}{a b}+\frac{w-w^{0}}{a(a-b)\left(w-e^{-a T} w^{0}\right)}+\frac{w-w^{0}}{b(b-a)\left(w-e^{-b T} w^{0}\right)} \mid$ & $\frac{1}{b-a}\left(\frac{e^{-\mu a T} w}{w_{d}-e^{-a T}}-\frac{e^{-\mu b T} w}{w_{d}-e^{-b T}}\right)$ \\
\hline$\frac{s+k}{(s+a)(s+b)}$ & $\frac{k}{a b}+\frac{k-a}{a(a-b)} \frac{w-w^{0}}{w-e^{-a T} w^{0}}+\frac{k-b}{b(b-a)} \frac{w-w^{0}}{w-e^{-b T} w^{0}}$ & $\frac{k-a}{b-a} \frac{e^{-\mu a T} w}{w_{d}-e^{-a T}}-\frac{k-b}{b-a} \frac{e^{-\mu b T} w}{w_{d}-e^{-b T}}$ \\
\hline$\frac{1}{s^{2}(s+a)}$ & $\begin{array}{l}{ }^{1}-+\left(\frac{T^{2}}{2 a}-\frac{T}{a^{2}}\right) \frac{w^{0}}{w-w^{0}} \\
+\frac{T^{2}}{a} \frac{\left(w^{0}\right)^{2}}{\left(w-w^{0}\right)^{2}}-\frac{1}{a^{3}} \frac{w-w^{0}}{w-e^{-a T} w^{0}}\end{array}$ & $\begin{array}{l}\frac{1}{a^{2}} \frac{(\mu a T-1) w}{w_{d}-1}+\frac{1}{a} \frac{T w}{\left(w_{d}-1\right)^{2}} \\
+\frac{1}{a^{2}} \frac{e^{-\mu a T} w}{w_{d}-e^{-a T}}\end{array}$ \\
\hline$\frac{s+k}{s^{2}(s+a)}$ & $\begin{array}{l}\frac{k}{a^{3}}-\frac{1}{a^{2}}+\left(\frac{T}{a}+\frac{k T^{2}}{2 a}-\frac{k T}{a^{2}}\right) \frac{w^{0}}{w-w^{0}} \\
+\frac{k T^{2}}{a} \frac{\left(w^{0}\right)^{2}}{\left(w-w^{0}\right)^{2}}+\left(\frac{1}{a^{2}}-\frac{k}{a^{3}}\right) \frac{w-w^{0}}{w-e^{-a T} w^{0}}\end{array}$ & $\begin{array}{l}\frac{k \mu a T+a-k}{a^{2}} \frac{w}{w_{d}-1}+\frac{k}{a} \frac{T w}{\left(w_{d}-1\right)^{2}} \\
+\frac{k-a}{a^{2}} \frac{e^{-\mu a T} w}{w_{d}-e^{-a T}}\end{array}$ \\
\hline
\end{tabular}




\begin{tabular}{|c|c|c|}
\hline$G(s)$ & $G_{p}(w)$ & $G(w, \mu)$ \\
\hline$\frac{s^{2}+k_{1} s+k_{0}}{s^{2}(s+a)}$ & $\left|\begin{array}{l}\frac{k_{0}}{a^{3}}-\frac{k_{1}}{a^{2}}+\left(\frac{k_{1} T}{a}+\frac{k_{0} T^{2}}{2 a}-\frac{k_{0} T}{a^{2}}\right) \frac{w^{0}}{w-w^{0}}+\frac{k_{0} T^{2}}{a} \\
\times \frac{\left(w^{0}\right)^{2}}{\left(w-w^{0}\right)^{2}}+\left(\frac{k_{1}}{a^{2}}-\frac{k_{0}}{a^{3}}\right) \frac{w-w^{0}}{w-e^{-a T} w^{0}}+\frac{1}{a} \frac{\left(1-e^{-a T}\right) w^{0}}{w-e^{-a T} w^{0}}\end{array}\right|$ & $\begin{array}{l}\frac{k_{0} \mu a T+k_{1} a-k_{0}}{a^{2}} \frac{w}{w_{d}-1}+\frac{k_{0}}{a} \frac{T w}{\left(w_{d}-1\right)^{2}} \\
+\frac{a^{2}-k_{1} a+k_{0}}{a^{2}} \frac{e^{-\mu a T} w}{w_{d}-e^{-a T}}\end{array}$ \\
\hline$\frac{1}{s(s+a)^{2}}$ & $\begin{array}{l}-\frac{2}{a^{3}}+\frac{1}{a^{2}} \frac{T w^{0}}{w-w^{0}}+\frac{2}{a^{3}} \frac{w-w^{0}}{w-e^{-a T} w^{0}} \\
+\frac{1}{a^{2}} \frac{T e^{-a T} w^{0}\left(w-w^{0}\right)}{\left(w-e^{-a T} w^{0}\right)^{2}}\end{array}$ & $\begin{array}{l}\frac{1}{a^{2}} \frac{w}{w_{d}-1}-\frac{1+\mu a T}{a^{2}} \frac{e^{-\mu a T} w}{w_{d}-e^{-a T}} \\
-\frac{1}{a} \frac{T e^{-(1+\mu) a T} w}{\left(w_{d}-e^{-a T}\right)^{2}}\end{array}$ \\
\hline$\frac{s+k}{s(s+a)^{2}}$ & $\begin{array}{l}-\frac{2 k}{a^{3}}+\frac{k}{a^{2}} \frac{T w^{0}}{w-w^{0}}+\frac{2 k}{a^{3}} \frac{w-w^{0}}{w-e^{-a T} w^{0}} \\
+\frac{1}{a^{2}} \frac{\left(1-e^{-a T}\right) w^{0}}{w-e^{-a T} w^{0}}+\frac{k-a}{a^{2}} \frac{T e^{-a T} w^{0}\left(w-w^{0}\right)}{\left(w-e^{-a T} w^{0}\right)^{2}}\end{array}$ & $\begin{array}{l}\frac{1}{a^{2}} \frac{k w}{w_{d}-1}+\frac{e^{-\mu a T}}{a^{2}} \frac{[\mu a T(k-a)-k] w}{w_{d}-e^{-a T}} \\
+\frac{e^{-(1+\mu) a T}}{a^{2}} \frac{(k-a) a T w}{\left(w_{d}-e^{-a T}\right)^{2}}\end{array}$ \\
\hline$\frac{1}{s(s+a)(s+b)}$ & $\begin{array}{l}-\frac{a+b}{a^{2} b^{2}}+\frac{T}{a b} \frac{w^{0}}{w-w^{0}}+\frac{1}{a^{2}(b-a)} \frac{w-w^{0}}{w-e^{-a T} w^{0}} \\
+\frac{1}{b^{2}(a-b)} \frac{w-w^{0}}{w-e^{-b T} w^{0}}\end{array}$ & $\begin{array}{l}-\frac{1}{a b} \frac{w}{w_{d}-1}+\frac{1}{a(a-b)} \frac{e^{-\mu a T} w}{w_{d}-e^{-a T}} \\
+\frac{1}{b(b-a)} \frac{e^{-\mu b T} w}{w_{d}-e^{-b T}}\end{array}$ \\
\hline$\frac{s+k}{s(s+a)(s+b)}$ & $\begin{array}{l}\frac{1}{a b}-\frac{(a+b) k}{a^{2} b^{2}}+\frac{k T}{a b} \frac{w^{0}}{w-w^{0}}+\frac{k-a}{a^{2}(b-a)} \frac{w-w^{0}}{w-e^{-a T} w^{0}} \\
\quad+\frac{k-b}{b^{2}(a-b)} \frac{w-w^{0}}{w-e^{-b T} w^{0}}\end{array}$ & $\begin{array}{l}\frac{k}{a b} \frac{w}{w_{d}-1}+\frac{k-a}{a(a-b)} \frac{e^{-\mu a T} w}{w_{d}-e^{-a T}} \\
+\frac{k-b}{b(b-a)} \frac{e^{-\mu b T} w}{w_{d}-e^{-b T}}\end{array}$ \\
\hline$\frac{s^{2}+k_{1} s+k_{0}}{s(s+a)(s+b)}$ & \begin{tabular}{|l}
$\quad \frac{k_{1}}{a b}-\frac{(a+b) k_{0}}{a^{2} b^{2}}+\frac{k_{0} T}{a b} \frac{w^{0}}{w-w^{0}}$ \\
$+\frac{a^{2}-k_{1} a+k_{0}}{a^{2}(b-a)} \frac{w-w^{0}}{w-e^{-a T} w^{0}}+\frac{b^{2}-k_{1} b+k_{0}}{b^{2}(a-b)} \frac{w-w^{0}}{w-e^{-b T} w^{0}}$
\end{tabular} & $\begin{array}{l}\frac{k_{0}}{a b} \frac{w}{w_{d}-1}+\frac{a^{2}-k_{1} a+k_{0}}{a(a-b)} \frac{e^{-\mu a T} w}{w_{d}-e^{-a T}} \\
+\frac{q^{2}-k_{1} b+k_{0}}{b(b-a)} \frac{e^{-\mu b T} w}{w_{d}-e^{-b T}}\end{array}$ \\
\hline$\frac{1}{(s+a)(s+b)(s+c)}$ & $\begin{array}{c}\frac{1}{a b c}-\frac{1}{a(b-a)(c-a)} \frac{w-w^{0}}{w-e^{-a T} w^{0}}-\frac{1}{b(a-b)(c-b)} \\
\times \frac{w-w^{0}}{w-e^{-b T} w^{0}}-\frac{1}{c(a-c)(b-c)} \frac{w-w^{0}}{w-e^{-c^{T}} w^{0}}\end{array}$ & $\begin{array}{l}\frac{1}{(b-a)(c-a)} \frac{e^{-\mu a T} w}{w_{d}-e^{-a T}}+\frac{1}{(a-b)(c-b)} \\
\times \frac{e^{-\mu b T} w}{w_{d}-e^{-b T}}+\frac{1}{(a-c)(b-c)} \frac{e^{-\mu c T} w}{w_{d}-e^{-c T}}\end{array}$ \\
\hline$\frac{s+k}{(s+a)(s+b)(s+c)}$ & $\mid \begin{array}{c}\frac{k}{a b c}-\frac{k-a}{a(b-a)(c-a)} \frac{w-w^{0}}{w-e^{-a T} w^{0}}-\frac{k-b}{b(a-b)(c-b)} \\
\times \frac{w-w^{0}}{w-e^{-b T} w^{0}}-\frac{k-c}{c(a-c)(b-c)} \frac{w-w^{0}}{w-e^{-c T} w^{0}}\end{array}$ & $\begin{array}{l}\frac{k-a}{(b-a)(c-a)} \frac{e^{-\mu a T} w}{w_{d}-e^{-a T}}+\frac{k-b}{(a-b)(c-b)} \frac{e^{-\mu b T} w}{w_{d}-e^{-b T}} \\
+\frac{k-c}{(a-c)(b-c)} \frac{e^{-\mu c T} w}{w_{d}-e^{-c T}} .\end{array}$ \\
\hline
\end{tabular}

